UCRL-JC-128812

PREPRINT

\title{
Multilayer Ultra High Gradient Insulator Technology
}

\author{
S. E. Sampayan \\ P. A. Vitello \\ M. L. Krogh \\ J. M. Elizondo
}

This paper was prepared for submittal to the

International Symposium on Discharges and Electrical Insulation in Vacuum Eindhoven, The Netherlands

August 17-21, 1998

March 27, 1998

This is a preprint of a paper intended for publication in a journal or proceedings. Since changes may be made before publication, this preprint is made available with the understanding that it will not be cited or reproduced without the permission of the author. 


\section{DISCLAIMER}

This document was prepared as an account of work sponsored by an agency of the United States Government. Neither the United States Government nor the University of California nor any of their employees, makes any warranty, express or implied, or assumes any legal liability or responsibility for the accuracy, completeness, or usefulness of any information, apparatus, product, or process disclosed, or represents that its use would not infringe privately owned rights. Reference herein to any specific commercial product, process, or service by trade name, trademark, manufacturer, or otherwise, does not necessarily constitute or imply its endorsement, recommendation, or favoring by the United States Government or the University of California The views and opinions of authors expressed herein do not necessarily state or reflect those of the United States Government or the University of California, and shall not be used for advertising or product endorsement purposes. 


\title{
MULTILAYER ULTRA-HIGH GRADIENT INSULATOR TECHNOLOGY
}

\author{
S. E. Sampayan, P. A Vitello, Lawrence Livermore National Laboratory, P.O Box 808, \\ Livermore, CA 94551, USA, M. L. Krogh and J. M. Elizondo, \\ AlliedSignal, FM\&T, P. O. Box 419159, Kansas City, MO 64141, USA
}

\begin{abstract}
We are investigating a novel insulator concept which involves the use of alternating layers of conductors and insulators with periods less than $1 \mathrm{~mm}$. These structures perform many times better (about 1.5 to 4 times higher breakdown electric field) than conventional insulators in long pulse, short pulse, and alternating polarity applications. We present our ongoing studies investigating the degradation of the breakdown electric field resulting from surface roughness, the effect of gas pressure, and the performance of the insulator structure under bi-polar stress. Further, we present our initial modeling studies.
\end{abstract}

\section{INTRODUCTION}

A high gradient insulator consists of a series of very thin $(<1 \mathrm{~mm})$ stacked laminations interleaved with conductive planes. This insulator technology was originally conceived and disclosed by Eoin Gray in the early 1980's [1] and follows from experimental observations that the threshold elcctric field for surface flashover increases with deceased insulator length [2, 3]. The concept laid dormant until the mid-to-late 1980 's', when this technology was pursued and successfully showed substantial increases in the breakdown threshold over conventional, single substrate insulators [4]. In more recent work, we pursued verification of the technology and showed an increase of 1.5 to 4.0 times that over conventional insulator technology [5] In later studies, we explored the properties of these structures in the context of switching applications, investigating their behavior under high fluence photon bombardment [6].

\section{EXPERIMENTS}

Small sample testing (approximately $2.5 \mathrm{~cm}$ diameter by $0.5 \mathrm{~cm}$ thick) was performed in a turbo-molecular pumped, stainless-steel chamber at approximately $10^{-6}$ T. High voltage was developed with a $10 \mathrm{~J}$ "miniMarx". The Marx developed a pulsed voltage of approximately 1 to $10 \mu$ s (base-to-base) and up to $250 \mathrm{kV}$ amplitude across the sample. Diagnostics consisted of an electric field sensor and a current viewing resistor. Failure of the insulator was determined by a prompt increase in Marx current and a prompt collapse in the voltage across the sample.

Samples were fabricated by interleaving layers of 0.25 $\mathrm{mm}$ fused silica. The interleaved metallic lavers were formed by depositing gold on each planar insulator surface by a sputtering technique and then bonding the stacked layers by heating while applying pressure. Bond strength between the gold layer and substrate using this technique was measured to exceed $10 \mathrm{kpsi}$. To perform the breakdown experiments, the structure was slightly compressed between highly polished bare aluminum electrodes which establish the electric field for the tests.

To obtain a particular data set, the insulators were subjected to several low voltage conditioning pulses. The voltage was then increased a small amount incrementally until breakdown occurred. Voltage was then reduced for several shots and then incrementally increased again until a constant value was achieved. In these experiments, however, we generally observed that these insulators did not condition. Once a breakdown occurred at a particular ficld, rcducing the voltage slightly and increasing it again did not cause an increase in brcakdown ficld To produce a given data set we would apply up to $150-200$ shots to a given structure and would attempt to determine if any damage to the structure occurred which significantly altered the breakdown characteristics At these applied energics, we generally did not observe any degradation. These data were then reduced to reliability plots by determining the total number of successful shots over the total number of applied shots. In these data we define the electric field as the applied voltage divided by the total insulator length. We define reliability at a given electric field as the total number of successful shots over the total number of shots.

Using this method, we observed flashover of the small samples at approximately $175 \mathrm{kV} / \mathrm{cm}$ for these fused silica substrates (Fig. 1). The effect of pulse width from 1-10 $\mu$ s on this breakdown threshold was well within the statistical nature of our data. The trend in conventional insulator technology (Fig 2) for $0^{\circ}$ insulators indicates a breakdown threshold of approximately 50 $\mathrm{kV} / \mathrm{cm}$. Thus, there was a net increase in the performance with these insulators over conventional technology of approximately 3.5 .

We are also studying various effects which can adversely affect these new structures. For instance, to ensure concentricity, a finish grinding operation was performed on the outside diameter. This process is a time consuming second operation and an alternate 
on the multilayer high gradient structures. Similar results are obtained for bi-polar pulses (Figure 6). This result leads us to conclude that this lamination technique scales the performance of thin insulators to the entire structure with each layer acting independently.

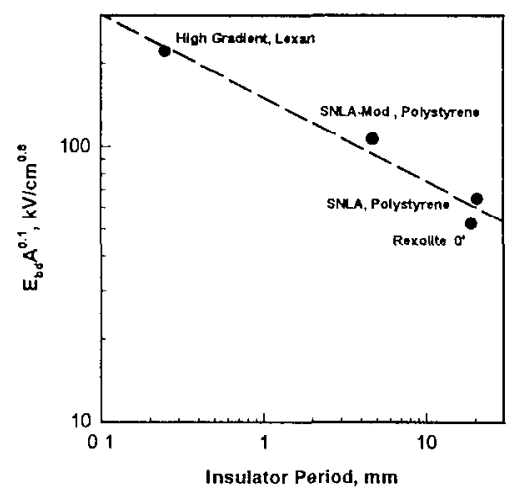

Fig. 6 The effect of period length scaling under bipolar stress [8]

\section{MODELING}

Voltage breakdown for insulators surrounded by vacuum is believed to take place along the insulator surface and not within the insulator or exterior to the insulator in the vacuum. Breakdown occurs on the time scale of nanoseconds, making this a difficult process to study experimentally. The basic physical processes involved are poorly understood Present theories of surface breakdown are mainly descriptive, and are not suited for the purpose of designing insulators. Due to the computationally intensive nature of a selfconsistently model of surface breakdown, few attempts have been made to model the complete process.

The two most widely accepted published models for surface breakdown focus on the initial process occurring either just below or just above the insulator surface $[9,10]$ These models focus respectively on solid state physics phenomena, and the propagation and emission of electrons through the vacuum just exterior to the insulator surface. Both models lead to surface heating, and evaporation of gas from the insulator. This evaporated gas is the medium where ultimately the voltage breakdown occurs along very localized "streamer" channels. Pressures in the evaporated gas close to the insulator surface where the discharges form can reach a significant fraction of atmospheric pressure. The surface breakdown is likely to be closely related to high pressure voltage breakdown processes [11].

We have compared processes involved in surface voltage breakdown with other well understood voltage breakdown phenomena at high pressure. Our conclu- sion is that an accurate theoretical model of surface breakdown must also include the long time scale evolution of the streamer discharge though the evaporated gas. Further, the tip of a propagating streamer is known to produce intense high energy radiation emission which we believe can lead to photo-conduction in the insulator. As conduction in the insulator will strongly modify the voltage which drives the streamer discharge, accurate coupling of the streamer to the insulator must also be included. Thus, our modeling approach is that the surface breakdown couples competing processes inside and external to the dielectric surface, and that a detailed, self-consistent model must be built to accurately study this process

Our baseline code is INDUCT95 [12] This code is a plasma discharge code that has been applied to plasma reactors, flat panel plasma display discharges, streamer discharges and other problems involving complex systems. It solves the standard time dependent fluid equations of the form

$$
\frac{\partial n_{1}}{\partial t}=-\nabla \cdot n_{1} \mathbf{v}_{1}+\sum_{j=1}^{N c} K_{i j}
$$

and:

$\frac{\partial h_{i} \mathbf{v}_{i}}{a}=-\nabla\left(n_{i} \mathbf{v}_{i} \quad \mathbf{v}_{i}\right)+\frac{q_{i} n_{i} \mathbf{E}}{m_{i}}-\frac{1}{m_{i}} \nabla n_{i} k T_{i}-\sum_{j=1}^{N_{N}} n_{i} \mathbf{v}_{i} v_{i j}(2)$ for the ions, where $R_{i}$ is the source term for the changes in the ion densities. And for the electrons

$$
\frac{\partial n_{e}}{\partial t}=-\nabla \quad \Gamma_{e}+\sum_{j=1}^{N_{e}} R_{e j}
$$

with the energy balance equation.

$$
\frac{\partial W_{e}}{\partial t}=-\nabla \cdot \mathbf{Q}-e \Gamma_{e} \cdot \mathbf{E}-P_{\text {inelastic }}
$$

where $\Gamma_{e}$ is the electron flux and $P_{\text {inelastic }}$ is the energy loss ratc duc to inclastic collisions

Preliminary examples of results arc shown in Figurcs 7 and 8 This simulation was performed with the updated version of INDUCT95/HYBRID and uses a MontcCarlo treatment of electrons and a fluid treatment of ions to follow the discharge development. Several geometry configurations and surface materials were investigated. The standard configuration studied had an electrode separation of $1 \mathrm{~mm}$ with an applied voltage of $50 \mathrm{kV} / \mathrm{cm}$ An insulator with a dielectric constant, $\varepsilon_{\mathrm{T}}=15$ was placed between the electrodes The dielectric was considered to either have a flat surface (Figure 7) or a step discontinuity (Figure 8) halfway between the electrodes. Electrons were launched with random initial direction from the cathode triple point until they either struck the insulator or the anode. Upon striking the insulator, electrons generated secondary electrons.

The secondary emission yield is a strong function of incident electron energy Secondary yield profiles were 
fabrication means was pursued To simplify fabrication, we attempted an ultrasonic machining process. Although it was possible to fabricate the part in a single operation, the surface was left slightly rougher. Comparison of the breakdown characteristics of these samples showed significantly more scatter and on average a slightly decreased breakdown threshold of approximately $25 \%$ (Fig 3)

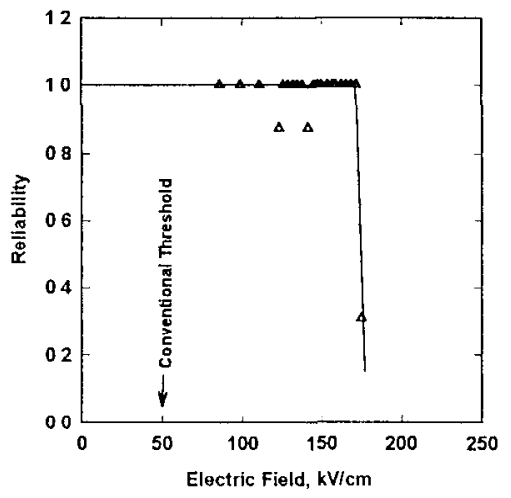

Fig 1 Pulsed surface breakdown reliability ground fused silica high gradient insulator

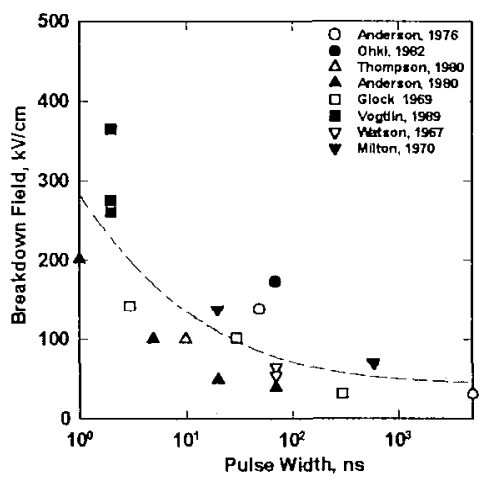

Fig 2. Pulsed surface breakdown electric field as a function of pulse width for single substrate, straight wall insulators

The structures were also subjected to increased pressures to determine susceptibility to breakdown (Fig. 4). In these data, using the previously described procedure, a fixed reliability was established at the various pressures All data was then normalized to a mean breakdown electric field. Susceptibility to breakdown stays relatively constant up until about the $10^{-3} \mathrm{~T}$ range, at which point, the field at which breakdown occurs decreases rapidly. Also shown are data from previous work by Smith [7]. From this comparison, it appears that these new structures show a lower breakdown electric field threshold than these previous data.

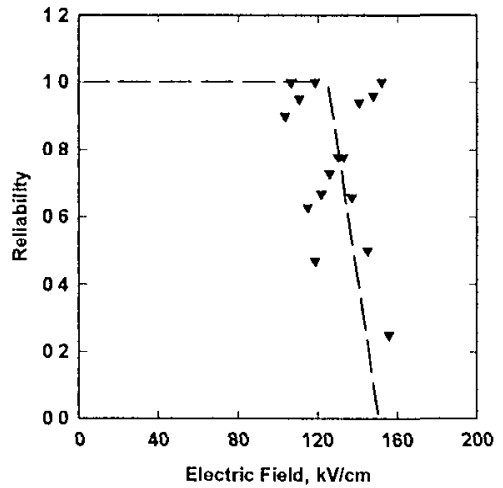

Fig 3 Pulsed surface breakdown reliability roughened fused silica high gradient insulator

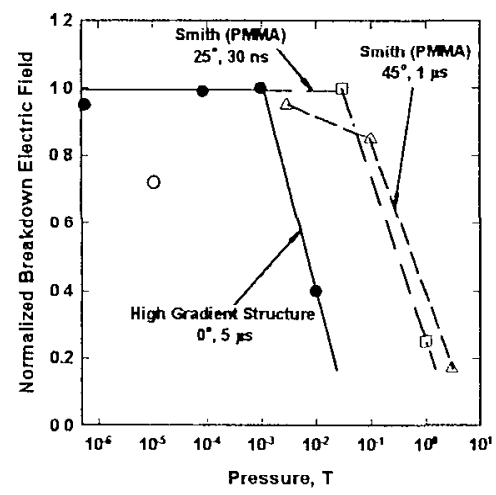

Fig. 4 Effect of gas pressure on the performance of high gradient insulators

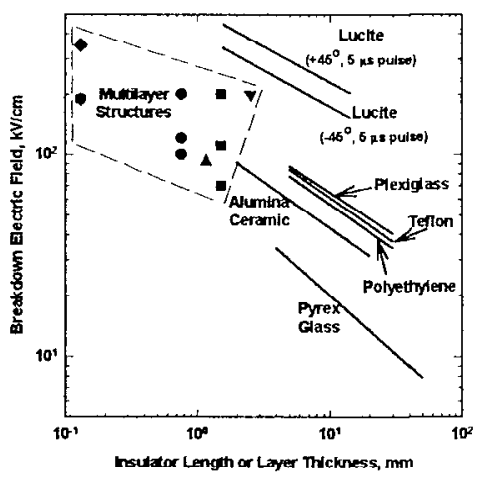

Fig. 5 The effect of insulator length scaling (conventional insulators, [3]) compared to individual layer thickness (multilayer insulators) on the surface breakdown electric field.

Our data for varied layer thickness and materials were compared to classical insulator length scaling (Figure 5 ). The trend is generally equivalent when the scale length is defined as the individual lamination thickness 
modeled for $\mathrm{Al}_{2} \mathrm{O}_{3}$ and $\mathrm{Cr}_{2} \mathrm{O}_{3}$ The secondary yield for $\mathrm{Al}_{2} \mathrm{O}_{3}$ has a maximum of $2+2$ while the $\mathrm{Cr}_{2} \mathrm{O}_{3}$ peaked at 0.98 . A yield greater than unity implies that the electron impact can lcad to a positive surface charge on the insulator as more than one electron will be emitted. Simulation results showcd strong positive surface charging for $\mathrm{Al}_{2} \mathrm{O}_{3}$ and weak negative surface charging for $\mathrm{Cr}_{2} \mathrm{O}_{3}$ for the flat diclectric casc (Figurc 7). Regions of positive surface charge on the insulator attract electrons cmittcd from the triple point and clcetrons cmitted by secondary emission. This attraction leads to enhanced scattering onto the insulator surface which results in avalanche. For $\mathrm{Cr}_{2} \mathrm{O}_{3}$, the negative surface charge repellcd clcetrons from the triple point, rcducing scattering and no avalanche was obtained. As would be expected, the insulator geometry was found to strongly influence surface charging A step dielectric geometry was also modeled for $\mathrm{Al}_{2} \mathrm{O}_{3}$. Strong ncgative surface charging developed along the step due to high energy electron impact (the secondary emission yicld drops below unity at high energy). This negative charge repelled electrons upwards away from the insulator surface, inhibiting the avalanche growth for this geometry.

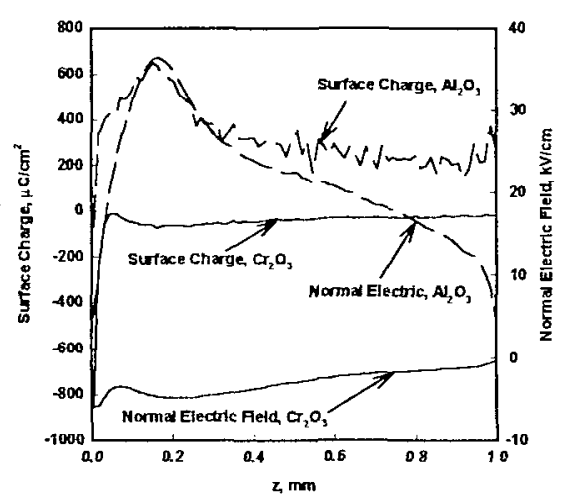

Fig. 7. INDUCT result for two secondary emission coefficients, straight insulator.

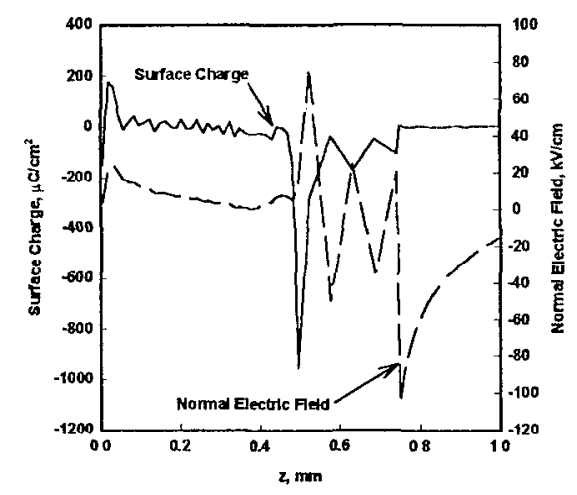

Fig. 8 INDUCT result for a straight insulator with an outward step discontinuity at $\mathrm{z}=0.5 \mathrm{~mm}$.

\section{SUMMARY}

We are continuing development of a new high gradient insulator technology. The insulator consists of finely spaced metal electrodes interleaved with the insulator substrate. We observe up to a factor of 4 improvement over conventional straight wall $\left(0^{\circ}\right)$ insulators We have also testcd the susceptibility of these insulators to breakdown under various conditions: surface roughness resulting from differcnt fabrication techniques and the effect of gas pressurc. Further, we are developing the necessary design tools to model and scale the bchavior of the high gradient insulator. From our study of present models, we conclude that an accurate thcorctical model of surface breakdown must also treat photoionization during the long time scalc evolution of the streamer discharge through the evaporated gas. That is, the tip of a propagating streamer is known to produce intense high energy radiation emission which we belicve can lead to photo-conduction in the insulator As conduction in the insulator will strongly modify the voltage which drives the streamer discharge, accurate coupling of the streamer to the insulator were included. We showed initial calculations that were performed

\section{REFERENCES}

a) Work performed under the auspices of the US Dept of Energy by LLNL under contract W-7405ENG-48

[1] Private communication, Eoin Gray

[2] O Milton, IEEE Trans. Electr Insul, vol EI-7, 1972, pp 9-15

[3] A. S Pillai and R. Hackam, J Appl Phys, vol 53(4), pp 2983-2987

[4] $J$ Elizondo and $\Lambda$ Rodriguez, in Proceedings of 1992 15th International Symposium on Discharges and Electrical Insulation in Vacuum (Vde-Verlag Gmbh, Berlin, 1992), pp. 198-202

[5] S. Sampayan, et al, in Proceedings of the 1995 Particle Accelerator Conference (IEEE, New York, NY, 1995), pp.1269-1271.

[6] S Sampayan, et al, in Proceedings of the 1995 Particle Accelerator Conference (IEEE, New York, NY, 995), pp 2123-2125.

[7] I. D Smith, "Pulsed Breakdown of Insulator Surfaces in Poor Vacuum," unpublished AWRE report, Aldermaston, United Kingdom

[8] R. A. Anderson and W. K Tucker, J.Appl.Phys, vol 58, pp 3346-3349 (1985)

[9] A. Watson, J. Appl. Phys, vol 38, p. 2019 (1967).

[10]G Blaise, J Appl Phys, vol. 77, 2916 (1995)

[11]E.W. Gray, J. Appl Phys., vol 58, 132-141(1985)

[12]P. A. Vitello, et.al., UCRL-MA120465, March 24, 1995 


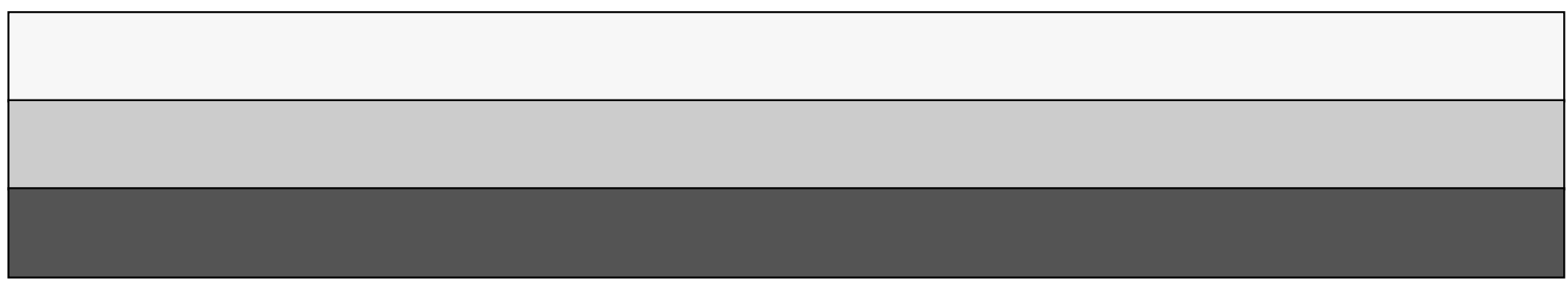

